rich air particles, as reported in steel production process, constitutes an important source of metal exposure, in particular to iron.

Methods We measured 8-OHdG in mitochondrial DNA (mtDNA), by real-time PCR, in blood leukocytes from 113 healthy male foundry workers (mean age $=42.2$ years, $\mathrm{SD}=$ 11.2) with high levels of exposure to metal-rich particles. Exposure to iron and others metals, was assessed in urine collected the same day of blood sampling, at the end of the standard working week. Multivariable regression models adjusted for age, body mass index (BMI), and smoking were designed to evaluate the relationship between urinary markers of exposure and 8OHdG in mtDNA To assure normal distribution, 8-OHdG in mtDNA data were $\log _{\mathrm{e}}$ transformed, and the regression slopes were exponentiated to obtain the geometric mean ratio (GMR) for increments in one SD of exposure.

Results After a week of exposure, elevated levels of urinary iron, (mean $\mathrm{Fe}=10.9 \mu \mathrm{g} / \mathrm{g}$ creat, $\mathrm{SD}=7.9$ ) were found among enrolled workers. Individual exposure level resulted positively associated with $8-\mathrm{OHdG}$ formation in mtDNA in peripheral blood leukocytes $(\mathrm{GMR}=1.22 \mathrm{p}=0.03)$. The observed association was confirmed also after adjustment for potential confounders: age, $\mathrm{BMI}$, and smoking $(\mathrm{GMR}=1.22 ; \mathrm{p}=0.04)$.

Conclusions Our observation of exposure-related high levels of 8-HOdG suggests that iron exposure may induce mtDNA damage, a potential response to oxidative stress caused by iron-induced production of ROS. The potential toxicity of high-level of iron exposure due to 8-HOdG generation and its ability to induce G-T base modification deserves further investigation.

\section{A TWO-YEAR FOLLOW-UP STUDY OF SALIVARY CORTISOL CONCENTRATION AND THE RISK OF DEPRESSION}

\begin{abstract}
${ }^{1} \mathrm{M}$ B Grynderup, ${ }^{2}$ Kolstad, ${ }^{3}$ Mikkelsen, ${ }^{4}$ Andersen, ${ }^{3}$ Bonde, ${ }^{5}$ Buttenschøn, ${ }^{4}$ Kærgaard, ${ }^{6}$ Kærlev, ${ }^{7}$ Rugulies, ${ }^{3}$ Thomsen, ${ }^{3}$ Vammen, ${ }^{5}$ Mors, ${ }^{8}$ Hansen. ${ }^{1}$ Aarhus University Hospital, Aarhus C, Denmark; ${ }^{2}$ Department of Occupational Medicine, Aarhus University Hospital, Aarhus, Denmark; ${ }^{3}$ Dep. of Occupational and Environmental Medicine, Bispebjerg University Hospital, Copenhagen, Denmark; ${ }^{4}$ Department of Occupational Medicine, Regional Hospital Herning, Herning, Denmark; ${ }^{5}$ Centre for Psychiatric Research, Aarhus University Hospital, Aarhus, Denmark; ${ }^{6}$ Center for National Clinical Databases South, Odense University Hospital, Odense, Denmark; ${ }^{7}$ National Research Centre for the Working Environment, Copenhagen, Denmark; ${ }^{8}$ Department of Public Health, University of Copenhagen, Copenhagen, Denmark
\end{abstract}

\subsection{6/oemed-2013-101717.397}

Objectives Demanding psychosocial working conditions are a suspected cause of depression. High cortisol concentration, a biomarker of an activated stress response, has been found in depressed patients. Increased physiological stress has been suggested as a mechanism linking psychosocial working conditions and depression. The aim of this study was to determine if a high level of salivary cortisol is a risk factor of depression.

Methods In 2007, we enrolled 4,467 public employees. Morning and evening salivary cortisol concentration were measured for each participant. Participants reporting high levels of depressive, burnout, or stress symptoms, assessed by questionnaires were assigned to a psychiatric interview. In this interview 98 participants were diagnosed with depression and subsequently excluded. Two years later in 2009, 2,920 participants who had provided at least one valid saliva cortisol measurement at baseline participated at follow up. The psychiatric interviews were repeated and 62 cases of newly onset depression were diagnosed. Odds ratios of depression were estimated for every $1.0 \mathrm{nmol} / \mathrm{l}$ increase in morning, evening, and daily mean cortisol concentration, as well as for the difference between morning and evening cortisol concentration.

Results The risk of depression decreased by increasing daily mean cortisol concentration and by increasing difference between morning and evening concentrations, while morning and evening cortisol concentrations were not significantly associated with depression. The adjusted odds ratios for $1.0 \mathrm{nmol} / \mathrm{l}$ increase in morning, evening, and mean daily cortisol concentration were 0.69 (95\% CI: 0.45-1.05), 0.87 (0.59-1.27), and 0.54 (0.32-0.90), respectively. The adjusted odds ratio for $1.0 \mathrm{nmol} / \mathrm{l}$ increase in difference between morning and evening concentration were $0.64(0.46-0.90)$.

Conclusions This study did not support the hypothesis that high salivary cortisol concentration is a risk factor of depression, but indicate that low mean salivary cortisol concentration and a small difference between morning and evening cortisol concentration may be risk factors of depression.

\section{OCCUPATIONAL NOISE EXPOSURE AND THE CORTISOL AWAKENING RESPONSE: THE IMPACT OF EXPOSURE LEVEL AND HEARING PROTECTION}

'Z A S Stokholm, ${ }^{2}$ Hansen, ${ }^{3}$ Bonde, ${ }^{4}$ Christensen, ${ }^{1}$ Frederiksen, ${ }^{5}$ Kristiansen, ${ }^{5}$ Lund, ${ }^{1}$ Vestergaard, ${ }^{6}$ Wetke, ${ }^{7}$ Kolstad. ${ }^{1}$ Aarhus University Hospital, Aarhus C, Denmark; ${ }^{2}$ Faculty of Health, University of Copenhagen, Copenhagen, Denmark; ${ }^{3}$ Department of Occupational and Environmental Medicine, Bispebjerg Hospital, Copenhagen, Denmark; ${ }^{4}$ Department of Internal Medicine and Cardiology A, Aarhus University Hospital, Aarhus, Denmark; ${ }^{5}$ National Research Centre for the Working Environment, Copenhagen, Denmark; ${ }^{6}$ Department of Audiology, University of Southern Denmark, Odense, Denmark; 'Department of Occupational Medicine, Aarhus University Hospital, Aarhus, Denmark

\subsection{6/oemed-2013-101717.398}

Objectives Environmental and occupational noise exposure have in some studies been related to increased risk of cardiovascular disease, hypothetically by activation of the hypothalamic-pituitary-adrenal (HPA) axis. The objective of this study was to investigate the relation between occupational noise exposure and the cortisol awakening response (CAR) as a measure of HPA activity. Methods This cross-sectional study included 398 industrial workers and as a reference 63 financial workers. Noise exposure levels were recorded every 5 seconds at the dominant shoulder by personal dosimeters for 24 hours and we calculated the $\mathrm{L}_{\text {Aeq }}$ value for work hours. For 310 workers who kept a diary on the use of hearing protection devices (HPD), we subtracted $10 \mathrm{~dB}$ from every noise recording obtained during HPD use and estimated the $\mathrm{L}_{\mathrm{AEq}}$ value at the ear. The next day salivary cortisol level was measured at awakening and after 10-60 min and the CAR was defined as the difference between the two.

Results The mean measured noise exposure level was $79.7 \mathrm{~dB}$ (A) [range: 55.0-94.2] and the mean estimated level at the ear $77.6 \mathrm{~dB}(\mathrm{~A})$ [range: 55.0-94.2]. In a linear regression model that adjusted for sex, age, calendar month, income, body mass index, sampling time and duration since occupational noise exposure, we observed no statistically significant exposure response relation between noise exposure level and CAR. This was neither the case in analyses of the effect of noise level estimated at the ear nor in internal analyses restricted to the industrial workers. Conclusions Neither measured nor estimated occupational noise exposure level was associated with the cortisol awakening response the following day. Thus, we found no indication that 